\title{
Radiologic Features of Distant Filler Migration with Inflammatory Reaction Following Augmentation Mammoplasty Using Aquafilling $®$
}

\section{Filler}

\author{
Kyung Hee Ko, ${ }^{1,}$ Hae Kyoung Jung, ${ }^{1}$ and Ah Young Park ${ }^{1}$ \\ ${ }^{1}$ Department of Radiology, CHA Bundang Medical Center, CHA University, School of Medicine, Seongnam-si, South Korea \\ "Corresponding author: Kyung Hee Ko, Department of Radiology, CHA Bundang Medical Center, CHA University, School of Medicine, 351 Yatapdong, Bundang-gu, Seongnam-si, \\ Gyeonggi-do, 463-712, South Korea. Tel: +82-317805498, E-mail: yourheeya@cha.ac.kr
}

Received 2016 November 29; Revised 2017 April 10; Accepted 2017 May 31.

\begin{abstract}
Historically, breast augmentation with injectable materials has been performed for decades. However, in the long term all materials led to unfavorable results with serious side effects. Recently, we came across a patient who had performed a breast augmentation using a brand-new Aquafilling® filler. Some insist that it is a very safe filler providing satisfactory improvement in breast shape and volume without any inflammatory reaction or serious adverse effect. Here, we present a 32-year-old woman who experienced severe complications 6 months after bilateral breast augmentation with Aquafilling® filler. The patient suffered from distant filler migration and inflammatory reaction extending from the left upper lateral abdominal wall to the vulva. Radiologic characteristics were very similar to those resulting from polyacrylamide gel (PAAG) mammoplasty. The use of Aquafilling® filler for breast augmentation should be strongly restricted until long-term safety is proved and verified.
\end{abstract}

Keywords: Augmentation Mammoplasty, Filler Migration, Hydrogel, Aquafilling®

\section{Introduction}

Breast augmentation with injectable material has been performed for decades. Historically, various materials have been used for injection, including paraffin, liquid silicone, and polyacrylamide gel (PAAG) (1). However, all materials resulted in complications such as pain, subcutaneous nodules, breast deformities and even loss of ability of breast feeding (2-4). For these reasons, U.S. food and drug administration (FDA) did not approve use of any kind of filler for breast augmentation.

Recently, we came across a patient who underwent breast augmentation using a brand-new Aquafilling® filler. Some plastic surgeons reported that Aquafilling® filler is a temporary filler providing satisfactory improvement in breast shape and volume without any inflammatory reactions or serious adverse effects (5). However, in this report, we present a 32-year-old woman who experienced severe complications 6 months after bilateral breast augmentation with Aquafilling® filler and discuss the radiologic characteristics.

\section{Case Presentation}

A 32-year-old woman presented with painful swelling on her left lower abdominal wall. She had received Aquafilling ${ }^{\circledR}$ filler injection for breast augmentation 6 months before at a plastic surgery clinic. She also complained of tenderness of her left breast with volume loss. The laboratory data showed increased levels of $C$ reactive protein (CRP) $(3.3 \mathrm{mg} / \mathrm{dL})$ and leukocytosis with neutrophilia (19920/uL, 89.3\%). Abdominal computed tomography (CT) showed low attenuated fluid collection in intermuscular and deep subcutaneous spaces with an accompanying ill-defined surrounding soft tissue infiltration spreading from the left upper lateral abdominal wall down to the lower anterior abdominal wall (Figure 1). Breast ultrasound revealed fluid like filler collection in a retroglandular, prepectoral location (Figure 2A). On the right, the large amount of filler collection was anechoic with internal multiple echogenic foci, while the collection on the left showed a marked decrease in volume, ill-defined margin, and heterogeneous internal echogenicity (Figure 2B). On Doppler, increased vascularity was noted on the left lower outer tender area (Figure $2 \mathrm{C}$ ). In addition, there were multiple, randomly distributed globules of the extravasated filler in bilateral breasts (Figure 2D). An incision was made in the affected lower abdominal wall and yellowish pus flowed out. Culture of the pus showed no bacterial growth. Nevertheless, she received intravenous antibiotic treatment for 10 days with percutaneous drainage of the abscess. The symptom gradually subsided.

One month later, she visited again for a painful swelling in her left vulvar area. On physical examination, 


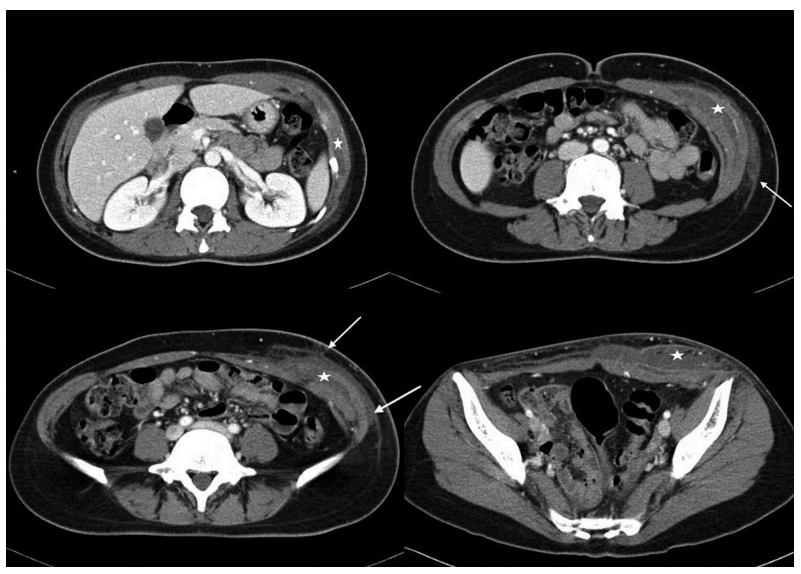

Figure 1. Radiologic features of abdominal computed tomography (CT). CT scan shows low attenuated fluid collection (star) in intermuscular and deep subcutaneous spaces with an accompanying ill-defined surrounding soft tissue infiltration (arrows) spreading from the left upper lateral abdominal wall down to the lower anterior abdominal wall.
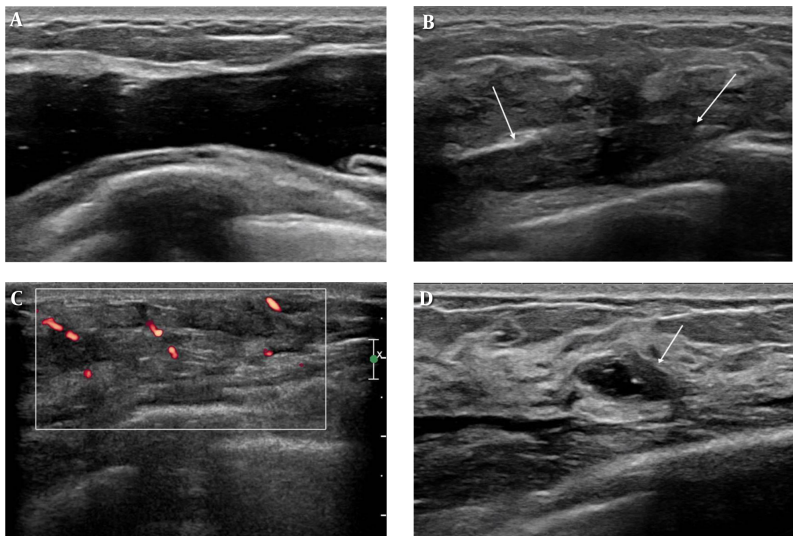

Figure 2. Breast ultrasound reveals fluid like filler collection in a retroglandular, prepectoral location. A, US of the right breast shows a large amount of anechoic filler collection with internal multiple echogenic foci. B, the left filler collection demonstrates a marked decrease in volume(arrows), ill-defined margin, and heterogeneous internal echogenicity. C, on Doppler, increased vascularity is noted at left lower outer tender area. D, Anechoic isolated filler collection (arrow) is noted at relative value scale update committee (RUC).

her left labium major showed severe redness and swelling and pus was oozing out from a fistula. Contrast-enhanced sagittal T1 weighted image (T1WI) demonstrated skin thickening and focal fluid collection with ill-defined reticular streaky soft tissue enhancement, extending from the left lower abdominal wall to the vulva (Figure 3 ). Clinician performed the incision and drainage for abscess and necrotic tissue from the left vulva. The patient has been treated for the lesion in the outpatient clinic for 6 months.

\section{Discussion}

Aquafilling® was developed in 2005 as soft tissue filler for facial contouring in Czech Republic and recently, it has been used for breast augmentation in several countries.

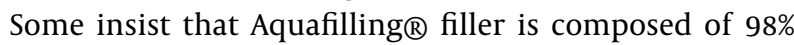
water and $2 \%$ copolyamide with excellent lifting capacity and viscoelasticity which can modify and maintain the breast form (5). However, in the document submitted to Korean food and drug administration (KFDA), the exact ingredient of Aquafilling $®$ filler is $2 \%$ of poly (acrylamide-coN,N-methylene-bis-acrylamide) and $98 \%$ of sodium chloride solution $0.9 \%$. In other words, the major component of Aquafilling® filler is polyacrylamide (6).

To the best of our knowledge, this is the first published case to report on the radiographic features of severe complications after augmentation mammoplasty using Aquafilling® injection. Unukovych et al. (7) re- 

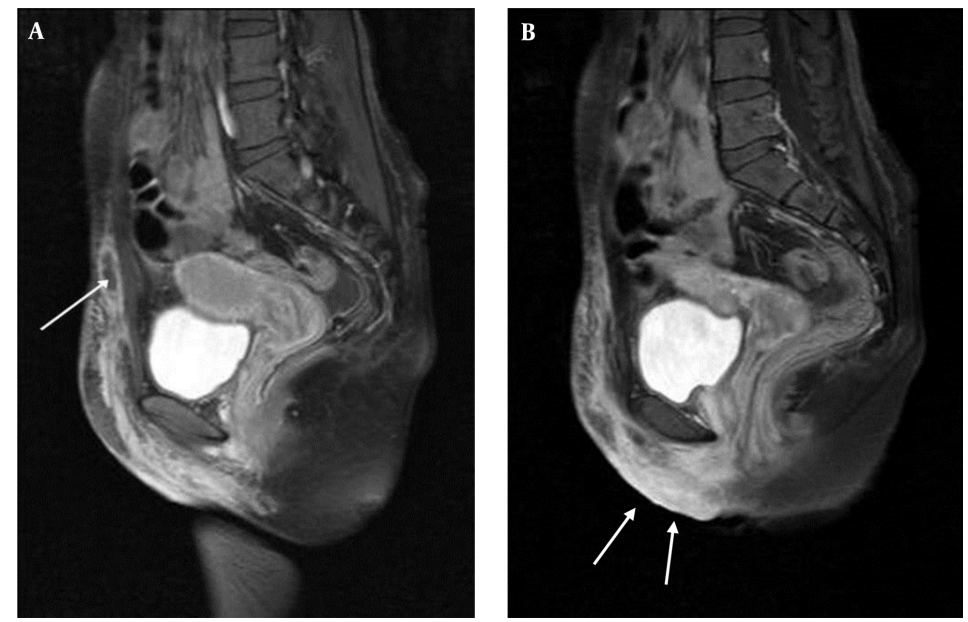

Figure 3. Radiologic features of abdominal magnetic resonance imaging (MRI). A and B, Contrast-enhanced sagittal T1 weighted image (T1WI) demonstrates skin thickening and focal fluid collection (arrow) with ill-defined reticular streaky soft tissue enhancement, extending from the left lower abdominal wall to the vulva (arrows).

ported about management of complications in 106 patients who had received PAAG injection for breast augmentation. The most common complications were breast pain (80\%), breast hardening and deformity (74\%), followed by gel migration (37\%). However, in that report, there was no patient who showed gel migration to the vulvar area, to say nothing of the fistula. According to previous studies, the mean time from the injection to complications was 51 months (range $=3-160)(8-10)$. The complications in this case occurred relatively early, just 6 months after the filler injection. We hypothesize that a large amount of injected filler or poor quality of procedure might cause the rapid onset of severe complications. Like as PAAG, Aquafilling® may absorb body fluid and exudate which could become a good medium for bacterial growth. In addition, it might also flow to a distant place under the influence of gravity, resulting in infection of loose connective tissues and fat layers (2). However, it would be necessary to clarify the exact mechanism of complication with more patients to judge the safety of Aquafilling® mammoplasty.

In this case, the radiologic evaluation was very useful in the investigation of a patient with complications after Aquafilling® mammoplasty. On ultrasound, uncomplicated Aquafilling® filler shows a large anechoic fluid collection with multiple internal echogenic foci. In the event of superimposed infection, the filler collection appears more heterogeneous and ill-defined. CT and MRI could precisely depict the extent of filler migration with adjacent soft tissue inflammatory changes. All of the radiologic findings in this case were very similar to those resulting from PAAG mammoplasty (11).

Polyacrylamide hydrogel (PAAG) was initially consid- ered to be a safe, non-toxic and non-allergenic and biocompatible. Since the discovery of PAAG, it has been widely used for breast augmentation as a minimally invasive technique. However, increasing serious complications after PAAG injection have been reported, for which many women suffered greatly $(3,8,10,12)$. PAAG is now prohibited for clinical use in most countries. Nevertheless today, more than 20 years after the introduction of PAAG, surgeons without any experience with these injections meet patients with PAAG related late complications (9). In order to avoid making the same terrible mistake, the use of Aquafilling® filler for breast augmentation should be strongly restricted until long-term safety is proved and verified.

In conclusion, we have shown the severe complications of bilateral breast augmentation with Aquafilling® injection. Radiologic features of them were very similar to those from PAAG mammoplasty. Knowledge of the radiologic characteristics of Aquafilling $®$ filler injected breasts as well as of related complications is very useful to make an accurate diagnosis and suggest proper management.

\section{References}

1. Peters W, Fornasier V. Complications from injectable materials used for breast augmentation. Can J Plast Surg. 2009;17(3):89-96. [PubMed: 20808751].

2. Wang ZX, Luo DL, Dai X, Yu P, Tao L, Li SR. Polyacrylamide hydrogel injection for augmentation mammaplasty: loss of ability for breastfeeding. Ann Plast Surg. 2012;69(2):123-8. doi: 10.1097/SAP.ob013e318225931c. [PubMed: 21785335].

3. Lee CJ, Kim SG, Kim L, Choi MS, Lee SI. Unfavorable findings following breast augmentation using injected polyacrylamide hydrogel. Plast Reconstr Surg. 2004;114(7):1967-8. [PubMed: 15577379]. 
4. Luo SK, Chen GP, Sun ZS, Cheng NX. Our strategy in complication management of augmentation mammaplasty with polyacrylamide hydrogel injection in 235 patients. J Plast Reconstr Aesthet Surg. 2011;64(6):731-7. doi: 10.1016/j.bjps.2010.10.004. [PubMed: 21074506].

5. Shin JH, Suh JS, Yang SG. Correcting Shape and Size Using Temporary Filler after Breast Augmentation with Silicone Implants. Arch Aesthet Plastic Surg. 2015;21(3):124. doi: 10.14730/aaps.2015.21.3.124.

6. Roh TS. Letter: Position Statement of Korean Academic Society of Aesthetic and Reconstructive Breast Surgery: Concerning the Use of Aquafilling $®$ for Breast Augmentation. Arch Aesthet Plastic Surg. 2016;22(1):45. doi:10.14730/aaps.2016.22.1.45.

7. Unukovych D, Khrapach V, Wickman M, Liljegren A, Mishalov V, Patlazhan $\mathrm{G}$, et al. Polyacrylamide gel injections for breast augmentation: management of complications in 106 patients, a multicenter study. World J Surg. 2012;36(4):695-701. doi: 10.1007/s00268-011-1273-6. [PubMed: 21932147].

8. Lin WC, Hsu GC, Hsu YC, Hsu HH, Li CS, Chen TY, et al. A late complication of augmentation mammoplasty by polyacrylamide hydrogel injection: ultrasound and magnetic resonance imaging find- ings of huge galactocele formation in a puerperal woman with pathological correlation. Breast J. 2008;14(6):584-7. doi: 10.1111/j.15244741.2008.00652.x. [PubMed: 19054003].

9. Patlazhan G, Unukovych D, Pshenisnov K. Breast reconstruction and treatment algorithm for patients with complications after polyacrylamide gel injections: a 10-year experience. Aesthetic Plast Surg. 2013;37(2):312-20. doi: 10.1007/s00266-012-0045-5. [PubMed: 23381651].

10. Cheng NX, Xu SL, Deng H, Ding XB, Zhang XM, Wu DH, et al. Migration of implants: a problem with injectable polyacrylamide gel in aesthetic plastic surgery. Aesthetic Plast Surg. 2006;30(2):215-25. doi: 10.1007/s00266-005-0081-5. [PubMed: 16547628].

11. Teo SY, Wang SC. Radiologic features of polyacrylamide gel mammoplasty. AJR Am J Roentgenol. 2008;191(3):W89-95. doi 10.2214/AJR.07.3616. [PubMed: 18716084].

12. Cheng NX, Liu LG, Hui L, Chen YL, Xu SL. Breast cancer following augmentation mammaplasty with polyacrylamide hydrogel (PAAG) injection. Aesthetic Plast Surg. 2009;33(4):563-9. doi: 10.1007/s00266008-9298-4. [PubMed: 19156460]. 